\title{
Leptons from decay of mesons in the laser-induced particle pulse from ultra-dense protium $\mathrm{p}(0)$
}

\author{
Leif Holmlid \\ Atmospheric Science, \\ Department of Chemistry and Molecular Biology, \\ University of Gothenburg, \\ SE-412 96 Göteborg, Sweden \\ holmlid@chem.gu.se
}

Received 16 June 2016

Revised 13 September 2016

Accepted 19 September 2016

Published 18 October 2016

\begin{abstract}
Kaons and pions are observed by their characteristic decay times of 12, 52 and 26 ns after impact of relatively weak ns-long laser pulses on ultra-dense hydrogen $\mathrm{H}(0)$, as reported previously. The signal using an ultra-dense protium $\mathrm{p}(0)$ generator with natural hydrogen is now studied. Deflection in a weak magnetic field or penetration through metal foils cannot distinguish between the types of decaying mesons. The signals observed are thus not caused by the decaying mesons themselves, but by the fast particles often at $>50 \mathrm{MeV} \mathrm{u}^{-1}$ formed in their decay. The fast particles are concluded to be mainly muons from their relatively small magnetic deflection and strong penetration. This is further supported by published studies on the direct observation of the beta decay of muons in scintillators and solid converters using the same type of $\mathrm{p}(0)$ generator.
\end{abstract}

Keywords: Ultra-dense hydrogen; mesons; leptons; nuclear processes.

PACS Number(s): 29.25.-t, 14.40.-n, 14.60.-x, 79.20.Ds

\section{Introduction}

Two different forms of ultra-dense hydrogen $\mathrm{H}(0)$ have been observed directly so far, namely ultra-dense protium $\mathrm{p}(0)^{1}$ and ultra-dense deuterium $\mathrm{D}(0){ }^{2,3}$ These materials are superfluid ${ }^{4,5}$ and also show a Meissner effect at room temperature, ${ }^{6,7}$ thus they are superconductive as well. The Meissner effect ${ }^{8}$ means that clusters of $\mathrm{H}(0)$ float in a magnetic field above the magnet. The superfluidity is observed both as fast diffusion over surfaces onto the outer surface of a container, ${ }^{6}$ and also as a so called fountain effect. ${ }^{4}$ The density of $\mathrm{H}(0)$ is close to $10^{29} \mathrm{~cm}^{-3}$ or $>100 \mathrm{~kg} \mathrm{~cm}^{-3} .9,10$ The

This is an Open Access article published by World Scientific Publishing Company. It is distributed under the terms of the Creative Commons Attribution 4.0 (CC-BY) License. Further distribution of this work is permitted, provided the original work is properly cited. 


\section{Holmlid}

most exact measurement of the normal distance D-D done on small clusters of the form $\mathrm{D}_{4}$ gives $2.15 \pm 0.02 \mathrm{pm},{ }^{11}$ while theory predicts $2.23 \mathrm{pm} .{ }^{3}$ Three different spin excitation levels have been observed, ${ }^{3}$ and the most common with $2.2-2.3 \mathrm{pm} \mathrm{D}-\mathrm{D}$ distance corresponds to $s=2 .^{9}$ The fundamental cluster structure of $\mathrm{H}(0)$ is given by chain clusters $\mathrm{H}_{2 N}$ with $N$ integer, formed by $\mathrm{H}-\mathrm{H}$ pairs rotating around the vortex. $^{4,12} \mathrm{H}(0)$ is not short-lived but can be retained on a suitable support for days and weeks in a vacuum. ${ }^{10}$

Nuclear fusion D + D giving both ${ }^{4} \mathrm{He}$ and ${ }^{3} \mathrm{He}$ was recently reported using $\mathrm{D}(0)$ on the laser target. ${ }^{13}$ Several studies show that massive particles (with mass) with high kinetic energy are ejected from $\mathrm{H}(0)$ by pulsed laser impact. The typical energy directly observed by time-of-flight (TOF) is around $10 \mathrm{MeV} \mathrm{u}^{-1}$, and up to $50 \mathrm{MeV} \mathrm{u} \mathrm{u}^{-1} \cdot{ }^{14-17}$ Normal $\mathrm{D}+\mathrm{D}$ fusion can only give particles with $3.0 \mathrm{MeV}$ $\mathrm{u}^{-1}$ in the first step, and in the second step of $\mathrm{T}$ or ${ }^{3} \mathrm{He}$ reacting with $\mathrm{D}$ the maximum kinetic energy is $14.7 \mathrm{MeV} \mathrm{u}^{-1}$. Thus, the nuclear processes observed are not normal $\mathrm{D}+\mathrm{D}$ nuclear fusion. It was shown that the time variation of the TOF signal agreed well with that expected for the formation and decay of particles with typical time constants in the range below $100 \mathrm{~ns}$, thus that the signal observed was due to intermediate particles in meson decay-chains. ${ }^{16,17}$ The decay time constants observed were 12, 26 and $52 \mathrm{~ns}$, thus the characteristic decay times for charged kaons, for pions and for neutral long-lived kaons. ${ }^{16,17}$ The remaining problem was mainly to understand if the mesons themselves or their product leptons (primarily muons) were detected by the collector at long distance from the laser target. This problem was recently further studied (submitted) by determining that the apparent TOF was not the true TOF, since the particles are often very fast and even relativistic. Here, we deflect the charged particles by magnetic fields and observe that there is no relation between the type and size of deflection and the decay life-time. The deflection observed agrees with light lepton decay products like muons and electrons. Thus, the meson decay takes place close to the laser target (the $\mathrm{H}(0)$ generator) and only the fast decay products pass through the deflecting magnetic field to be detected at the collector. These results may be of great importance for useful muon-catalyzed nuclear fusion. ${ }^{18,19}$

\section{Theory}

Ultra-dense hydrogen $\mathrm{H}(0)$ is a quantum material at room temperature. It is described in several publications. Detailed studies of the structure of $\mathrm{D}(0)^{3,9}$ and of $\mathrm{p}(0)^{1}$ have been made. The name was changed recently from $\mathrm{H}(-1)$ to $\mathrm{H}(0)$, since the material is not inverted (this assumption motivated the negative sign). Instead of inverted Rydberg matter, it is spin-based Rydberg matter ${ }^{3}$ with orbital angular momentum $l=0$ for the electrons. It is shown to be both superfluid ${ }^{4,6}$ and superconductive (Meissner effect ${ }^{8}$ observed) at room temperature. $^{6,7}$ The measured $\mathrm{H}-\mathrm{H}$ distances are short, normally 2.3 pm. ${ }^{1,3,9}$ Several spin states with different internuclear distances exist. ${ }^{3}$ It is likely that the main process initiated by the impinging 
laser pulse is a transition from level $s=2$ with $\mathrm{H}-\mathrm{H}$ distance of $2.3 \mathrm{pm}$, to level $s=1$ with theoretical distance $0.56 \mathrm{pm} .{ }^{3,20}$ At this distance, nuclear reactions are spontaneous and laser-induced nuclear processes are thus relatively easy to start. In muon catalyzed fusion, the D-D distance is of similar size, and the rate of fusion is close to $10^{9} \mathrm{~s}^{-1} \cdot{ }^{19}$ If a transition to level $s=1$ can take place spontaneously, a spontaneous nuclear process is possible, similar to the ones often named LENR. ${ }^{21}$ Several studies have proved the formation of $\mathrm{MeV}$ particles from $\mathrm{D}(0)$ during laser impact under conditions useful for ICF. ${ }^{14,22,23}$ Particle energies up to $50 \mathrm{MeV}^{-1}$ have been observed. ${ }^{14,15}$ Most high-MeV particles are neutral at least initially.

Ordinary Rydberg matter (orbital angular momentum $l$-based Rydberg matter) has $l>0$ for its binding electrons. ${ }^{24}$ Ultra-dense hydrogen has $l=0$ and $s>0$ $(1,2,3, \ldots)$, i.e., the spin quantum number for the bonding electrons. Thus, the electrons which give the ultra-dense matter structure have only a spin motion and no orbital motion. This electron spin motion may be interpreted as a motion of the charge with orbit radius $r_{q}=\hbar / 2 m_{e} c=0.192 \mathrm{pm}$ and with the velocity of light $c$ ('zitterbewegung'). ${ }^{25}$ This spin motion is centered on the $\mathrm{D}$ atoms and may give a planar structure for the D-D pairs as in the case of the planar clusters for ordinary Rydberg matter. This means that the interatomic distance in ordinary Rydberg matter which is $d=2.9 l^{2} a_{0}$ is replaced by $d=2.9 s^{2} r_{\mathrm{q}}$ for the ultra-dense matter. This was shown to be correct for $\mathrm{H}(0)$ by direct measurements. ${ }^{3}$ The Bohr radius is indicated as $a_{0}$. Here, 2.9 is a scaling constant determined numerically for ordinary Rydberg matter ${ }^{26}$ and confirmed experimentally for ordinary Rydberg matter by radio frequency spectroscopy. ${ }^{27,28}$

The mechanism for formation of ultra-dense matter starts with the formation of higher normal Rydberg matter levels of $\mathrm{H}_{N}(l \geq 1) \cdot{ }^{24}$ Such ordinary Rydberg matter is formed spontaneously at the iron oxide catalyst. $\mathrm{H}(0)$ is then formed spontaneously from the normal Rydberg matter. This mechanism for transfer to ultra-dense matter is depicted in Fig. 2 in Ref. 20. It implies that the ultra-dense type is formed from ordinary Rydberg matter levels falling down to the lower energy ultra-dense states. This process is even observed directly in the experiments as an oscillation in real time. ${ }^{10} \mathrm{H}(0)$ forms a layer on the catalyst and on the upper surface of the $\mathrm{H}(0)$ generator. The laser impact induces the nuclear processes which eject particles from this layer. These particles are deflected and detected in the apparatus.

The origin of the meson and lepton signal observed is clearly based on laserinduced nuclear processes in $\mathrm{H}(0)$. The laser energy induces a first step as a transfer of the $\mathrm{H}_{2}(0)$ pairs in the ultra-dense material $\mathrm{H}(0)$ from excitation state $s=2$ (with $2.3 \mathrm{pm} \mathrm{H}-\mathrm{H}$ distance) to $s=1$ (at $0.56 \mathrm{pm} \mathrm{H}-\mathrm{H}$ distance). ${ }^{3}$ In state $s=1$, the nuclei are so close that a tunneling and weak interaction may take place between two protons. The weak interaction does not conserve strangeness. ${ }^{29}$ Experiments show that the hydrogen atoms in state $s=1$ stay at this distance for a time longer than the laser pulse-length of $5 \mathrm{~ns},{ }^{3}$ thus much longer than the typical time for weak decay processes of $10^{-10} \mathrm{~s}^{29}$ From the six quarks in the two protons, three 


\section{Holmlid}

kaons are suggested to be formed in this interaction. Two protons have a mass of $1.88 \mathrm{GeV}$ while three kaons correspond to $1.49 \mathrm{GeV} .{ }^{30}$ Thus, a transition $2 \mathrm{p} \rightarrow$ $3 \mathrm{~K}$ may release $390 \mathrm{MeV}$. If only pions would be formed, which does not require a change to strange quarks, the excess energy is much larger. The charged and neutral kaons decay in various processes mainly to charged pions and muons. ${ }^{31}$ The decay of kaons and pions is observed directly through their decay to muons, while the muons may leave the chamber before they decay appreciably due to their much longer lifetime. As described below, they may interact with the chamber materials giving a shorter effective lifetime.

The decay-time experiments require some analysis. The signal is measured in a beam ejected from the pulsed-laser interaction with ultra-dense hydrogen $\mathrm{H}(0)$ on the generator. The signal is as shown previously ${ }^{16,17}$ due to an intermediate particle $\mathrm{M}$ which is formed and decays like $\mathrm{A} \rightarrow \mathrm{M} \rightarrow \mathrm{N}$. The time dependence of the signal $\mathrm{M}$ is easily derived from the rate equations for $\mathrm{A} \stackrel{k_{1}}{\rightarrow} \mathrm{M} \stackrel{k_{2}}{\rightarrow} \mathrm{N}^{16}$

$$
\begin{aligned}
-\frac{d n_{\mathrm{A}}}{d t} & =k_{1} n_{\mathrm{A}}, \\
\frac{d n_{\mathrm{M}}}{d t} & =k_{1} n_{\mathrm{A}}-k_{2} n_{\mathrm{M}},
\end{aligned}
$$

as

$$
n_{\mathrm{M}}=\frac{k_{1}}{k_{2}-k_{1}} n_{\mathrm{A} 0}\left(e^{-k_{1} t}-e^{-k_{2} t}\right),
$$

where $n_{\mathrm{A} 0}$ is the number density of the precursor at time $t=0$ thus during the laser pulse. Equation (3) assumes that the initial number density $n_{\mathrm{M} 0}$ is zero. The curve shape in Eq. (3) is used to match the decay results presented in the figures below. The results are given as time constants $\tau=1 / k$.

\section{Experimental}

The experimental setup with dimensions is shown in Fig. 1. The laser used is a Nd:YAG laser with pulse energy $<0.4 \mathrm{~J}$ at $1064 \mathrm{~nm}$ and pulse length $7 \mathrm{~ns}$. The pulse repetition rate is $10 \mathrm{~Hz}$. The laser beam was focused with an $f=50 \mathrm{~mm}$ lens on the $H(0)$ surface layer on a metal surface in a small vacuum chamber. The laser beam waist is $<20 \mu \mathrm{m}$ as calculated for a Gaussian beam. This means a laser intensity of $<4 \times 10^{13} \mathrm{~W} \mathrm{~cm}^{-2}$ for a Gaussian beam.

In the $\mathrm{H}(0)$ generator, several potassium doped iron oxide catalyst samples ${ }^{32,33}$ form $\mathrm{p}(0)$ from natural hydrogen gas (99.9995\% pure hydrogen, naturally containing only $0.016 \%$ D). Some of the ultra-dense material formed stays on the generator upper surface, where the laser interacts with it. The gas pressure in the chamber is $0.2-0.4$ mbar (uncorrected Pirani gauge reading) with constant pumping. The dividing wall shown in Fig. 1 has a $10 \mathrm{~mm}$ diameter circular aperture. It is either 1.2 or $4.2 \mathrm{~mm}$ thick stainless steel. The whole chamber is constructed in nonmagnetic stainless steel. 


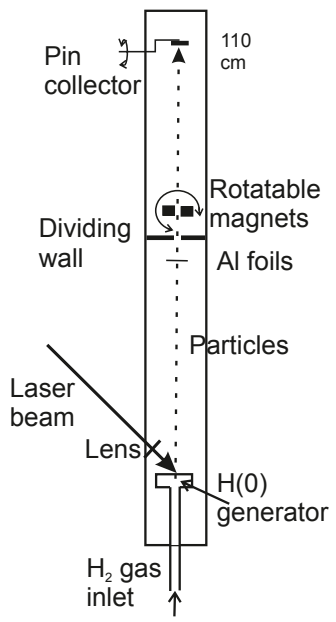

Fig. 1. Principle of the apparatus used, vertical cut. The pin collector can be moved on a circle with $3 \mathrm{~cm}$ radius. The inner diameter of the tube is $100 \mathrm{~mm}$. The $\mathrm{Al}$ foils shown in the beam are only used in a few experiments.

Some of the particles ejected from the $\mathrm{H}(0)$ generator by the laser pulse move through the opening in the dividing wall and can reach the pin collector. This pin is $1 \mathrm{~mm}$ wide and $20 \mathrm{~mm}$ long, mounted with an offset of $30 \mathrm{~mm}$ on a rotating feedthrough so that it can be moved sideways. It covers a fraction of $1.4 \times 10^{-6}$ of the entire $4 \pi$ sphere around the generator. In this way, the deflection of charged particles in the magnetic field can be measured. The magnetic field is due to two ferrite permanent magnets (Y28, $30 \times 10 \times 5 \mathrm{~mm}$ ) with a distance of $5 \mathrm{~mm}$ between their poles, and has a field strength of $100 \mathrm{mT}$. The magnets are mounted on a rotating and translating feed-through as shown in Fig. 2. The distance from the magnets to the pin collector is $40 \mathrm{~cm}$. The magnets can be rotated to block the beam or give alternate field directions and can be drawn sideways to give a negligible field at the beam passing through the opening in the dividing wall.

The current to the collectors is taken out via a short $50 \Omega$ coaxial cable to the $50 \Omega$ input of a fast digital two-channel oscilloscope (Tektronix TDS 3032, $300 \mathrm{MHz}$, $1.2 \mathrm{~ns}$ rise time). Thus, a signal of $1 \mathrm{~V}$ corresponds to $20 \mathrm{~mA}$ of current. A shielded $50 \mathrm{~V}$ battery can be inserted into the signal path at the feed-through in the vacuum wall to give positive or negative bias voltages on the collector, still giving a $50 \Omega$ connection to the oscilloscope. It introduces a delay of the signal of $2-3 \mathrm{~ns}$.

\section{Results}

The signal induced by the charge pulse from the generator is often large. Both the direct electromagnetic pulse to the collector and the pulse generated by the return current flowing in the tube-formed vacuum chamber give a substantial signal at the collector. This is demonstrated in Fig. 3, where an example of the observed 


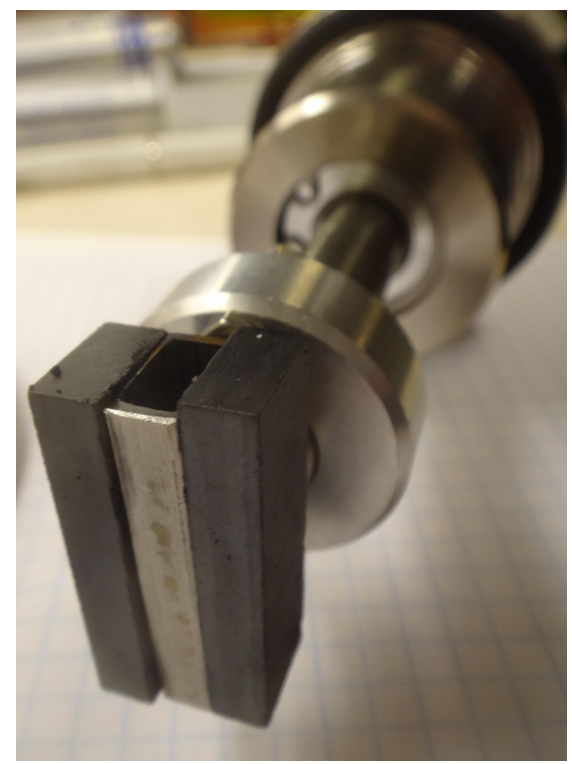

Fig. 2. Magnet holder, with ferrite magnet dimensions $30 \times 10 \times 5 \mathrm{~mm}$. The particles to the pin collector pass through the rectangular tube between the magnets.

signals is shown. The true particle signal is obtained after subtraction of the induced voltage signal measured at a location where the particle signal is small. The typical decay time constants for kaon and pion decay are shown after such subtraction in Figs. 4 and 5. Similar results have been published previously. ${ }^{16,17}$ Such decay curves may be (1) due to decaying mesons in the beam to the collector, or (2) due to decaying mesons at the generator, sending their decay products with high velocity to the collector. In case (1), the mesons are detected while their products are not observed, while in case (2), the mesons do not reach the collector and only their decay products with high velocity are observed at the collector. In case (1), the velocity of the mesons themselves may need to be so high that the transport time is much shorter than their life-time, but several complications will still exist in such a process. Thus, it is likely that case (2) is correct, and this point is investigated directly here.

No direct relation between the detection of the typical decay constants and the measurement parameters (like magnet (or) no magnet, $0 \mathrm{~V}$ (or) $-50 \mathrm{~V}$ bias voltage on the collector, central (or) deflected signal) has been found. This of course supports case (2) above, that the decaying mesons are relatively slow and that their fast moving decay products are the particles detected. The experiments give information also on other particles. For example, the final particles after muon decay will be electrons and positrons $\mathrm{e}^{ \pm}$. Examples of their detection are shown in Figs. 6 and 7 , using deflection only in the geomagnetic field. It is generally found that the signal without magnet is deflected from the center, thus indicating electrons 

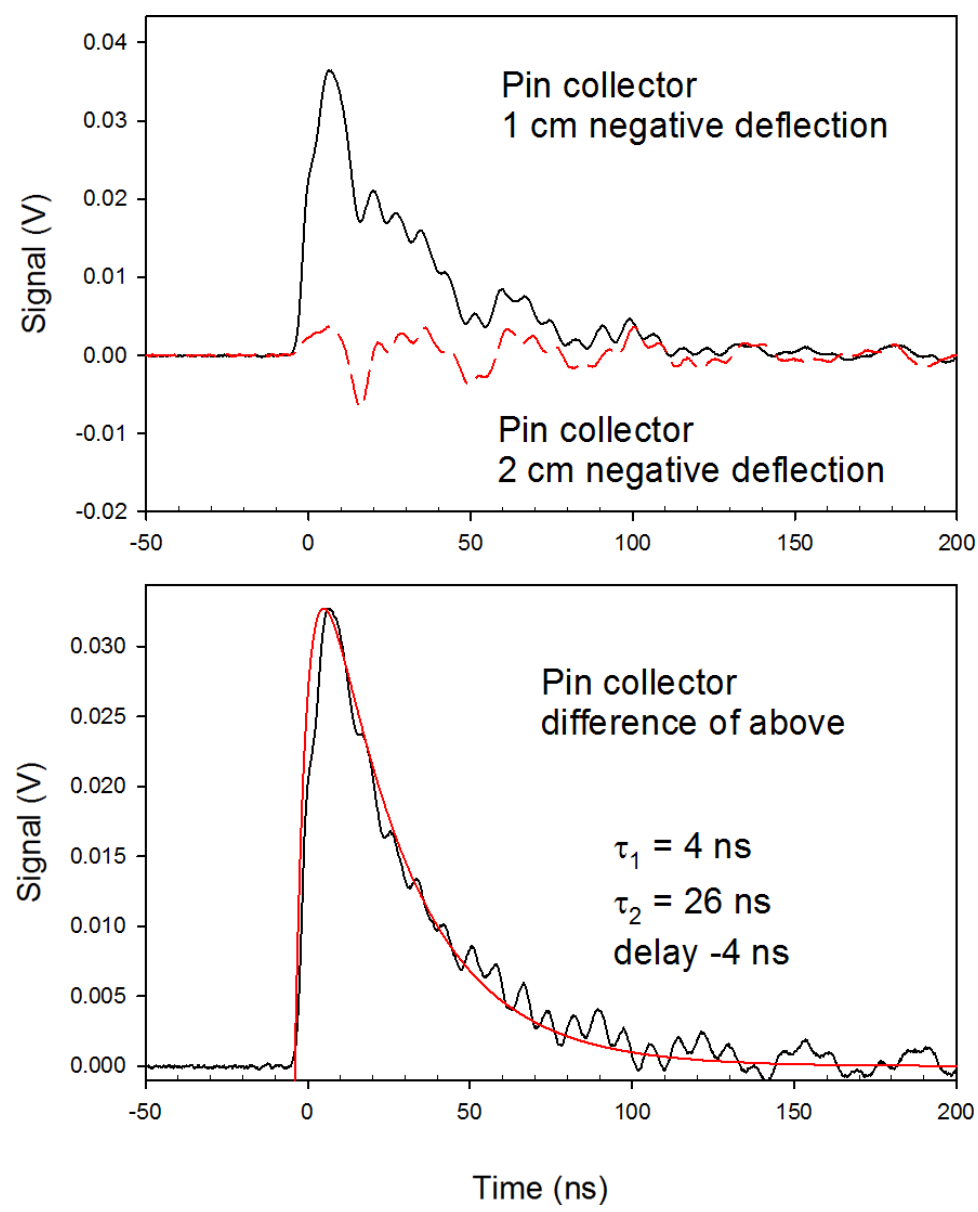

Fig. 3. Signal at pin collector, no magnet, zero bias. Upper panel shows two pin positions, lower panel shows difference signal and calculated exponential decay fit. Decay time-constant indicates $\pi^{ \pm}$decay.

and positrons in the case of only geomagnetic field. The deflection of $\mathrm{e}^{ \pm}$in the $0.1 \mathrm{~T}$ magnetic field used for deflecting mesons and muons is too large to be useful here (with particles $\mathrm{e}^{ \pm}$hitting the chamber wall). This particle deflection in the geomagnetic field means also that muons do not give the signal, since the deflection is too large for muons. The TOF of the signal in Fig. 6 is so short that a high velocity of the particles is likely. The size of the deflection on the other hand indicates a velocity as low as $20 \mathrm{MeV} \mathrm{u}^{-1}$ for electrons, thus an electron kinetic energy of $10 \mathrm{keV}$. This deflection may indicate thermal electrons from the fusion process (as observed previously at $500 \mathrm{MK}^{34}$ ) or electrons from pair production. ${ }^{35}$ The direct line-of-sight from the generator is not blocked in this experiment, so the fastest part of the signal could still be due to scattered photons, but the characteristic exponential form of the signal contradicts this. However, the most likely process for 


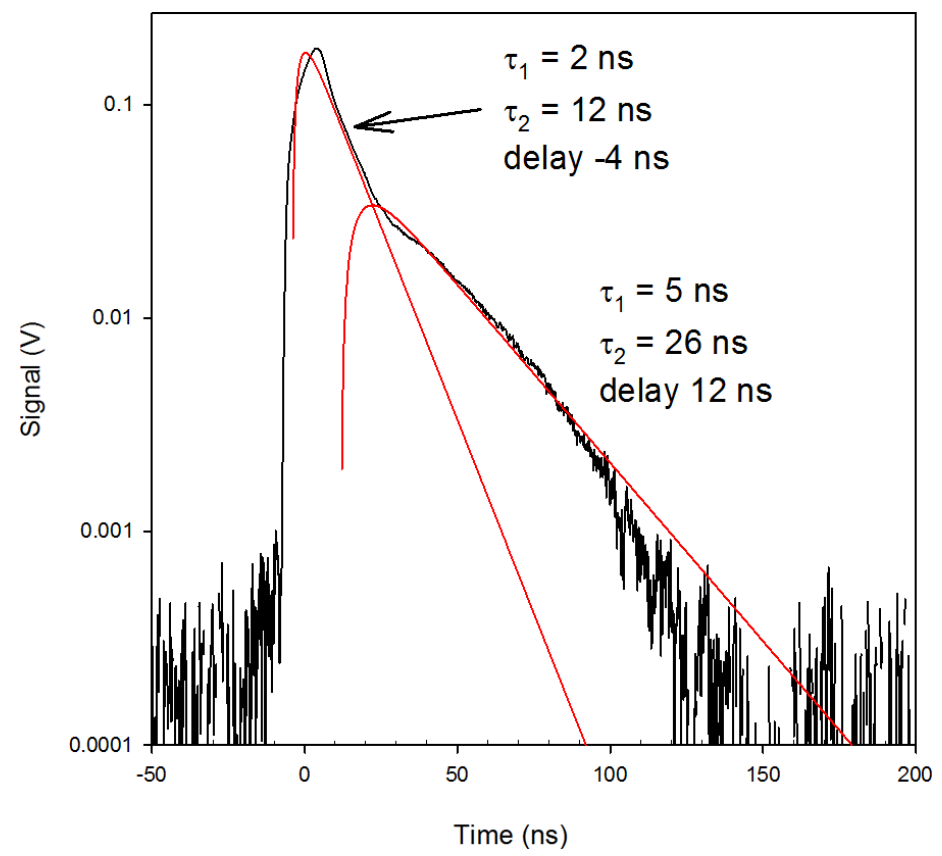

Fig. 4. Decay time-constants of $12 \mathrm{~ns}$ and $26 \mathrm{~ns}$, indicating $\mathrm{K}^{ \pm} \rightarrow \pi^{ \pm} \rightarrow \mu^{ \pm}$. Note that the delay for the second step is $12 \mathrm{~ns}$ as for the first decay step, as expected. Signal is difference negative deflection-positive deflection, negative bias, with magnet.

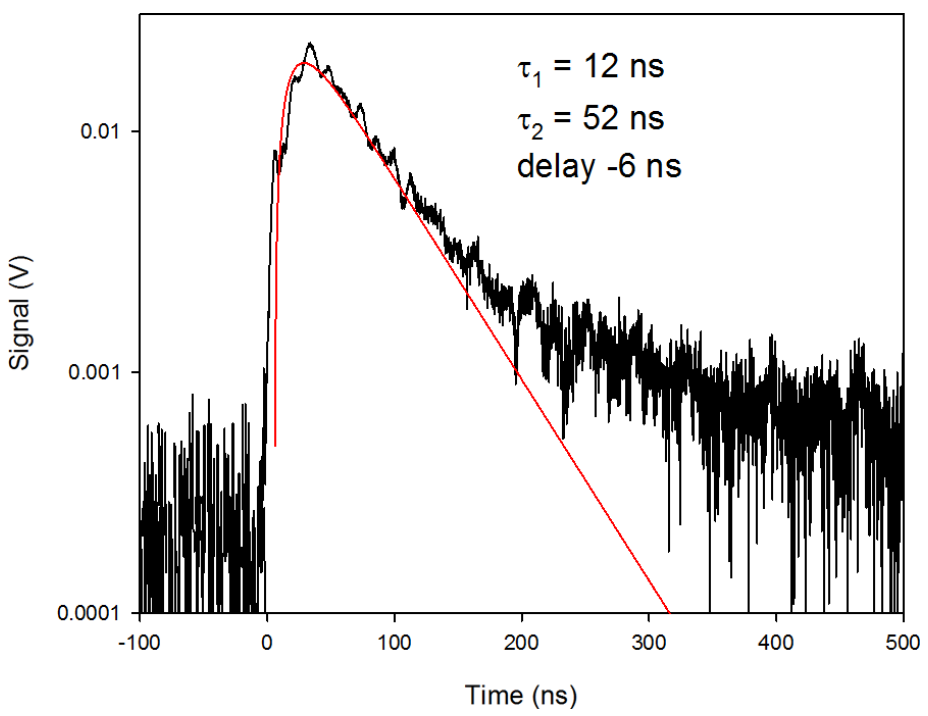

Fig. 5. Decay time-constant of $52 \mathrm{~ns}$ indicating $K_{L}^{0} \rightarrow$ decay. Signal is difference negative deflection-positive deflection, zero bias, with magnet. 


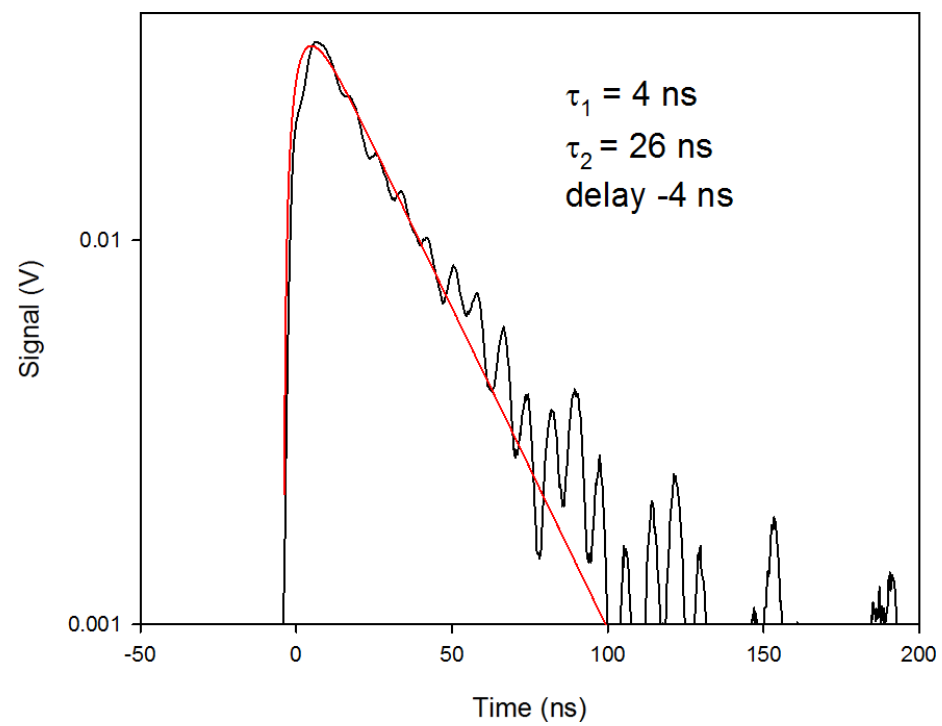

Fig. 6. Electrons as secondary signal. Decay time-constant indicates $\pi^{ \pm}$decay. Signal is difference negative deflection-center, zero bias, no magnet.

electron production here is secondary from the muons, thus in the case in Fig. 6 as $\pi^{-} \rightarrow \mu^{-} \rightarrow e^{-}$. The decay time of free negative muons is $2.2 \mu \mathrm{s}$, and the electron production should thus here instead be via formation of muonic atoms and muon capture. ${ }^{36}$ The reason why the signal is a positive current to the collector is that the energetic electrons eject secondary electrons from the collector which appear as a positive current to the collector, even with zero bias at the collector.

The sign of deflection in Fig. 7 instead indicates positive particles deflecting with no magnet (only the geomagnetic field). Thus, the particles may be positrons detected as a positive current. As in the case of the electron signal, the particle signal is expected to be mainly observed by secondary electrons ejected from the pin collector, thus giving a positive signal current. The complex signal decay shows two time constants, corresponding to $\mathrm{K}^{ \pm} \rightarrow \pi^{ \pm} \rightarrow \mu^{ \pm}$. The positive muons could then decay to $\mathrm{e}^{+}$. The decay time constant for muons is $2.2 \mu \mathrm{s}$, while the total elapsed time shown in Fig. 7 is only $200 \mathrm{~ns}$. However, since the production of the muons is observed, giving the decay time constants of the kaons and pions, the muons just interact with the collector pin and decay later at a longer distance. Thus, positive muon decay is not the process which gives the observed positron signal. In Fig. 7, the signal rise is slower than in Fig. 6, and a further delay is observed for the positive deflected signal. This indicates that this positron signal is due to other processes than muon decay, possibly pair production by interaction with the chamber walls. ${ }^{35}$

Magnetic deflection experiments at $0.1 \mathrm{~T}$ field strength give further information. One possibility is to measure with the magnetic field deflecting consecutively in 


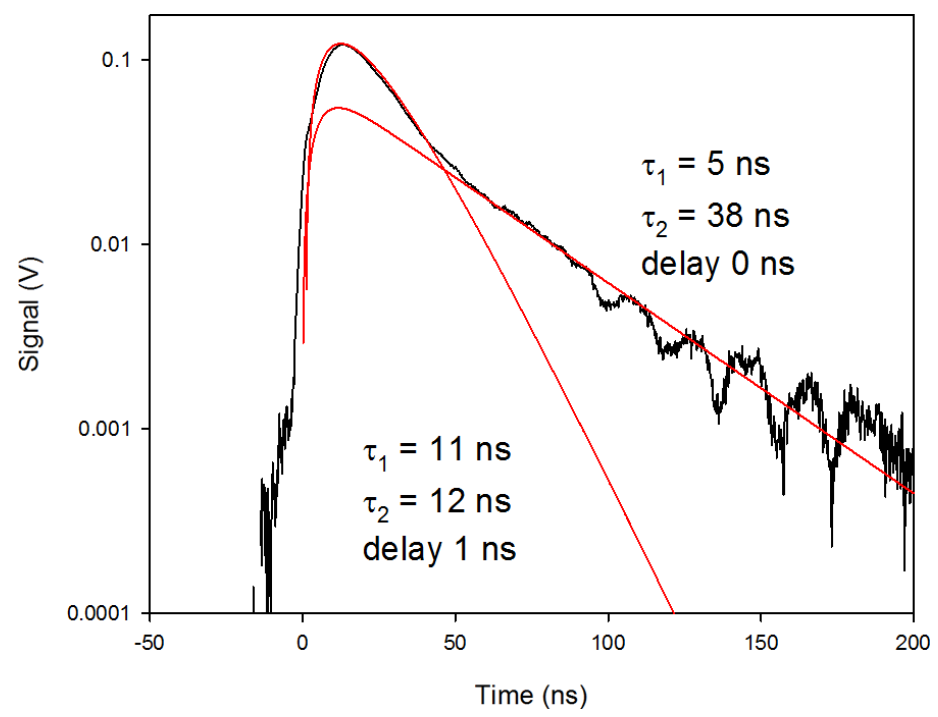

Fig. 7. Positrons as secondary signal. Decay time-constants indicate $\pi^{ \pm}$and combined $\mathrm{K}^{ \pm}+\pi^{ \pm}$ decay. Signal is difference no magnet-magnet, zero bias, positive deflection.

both the directions, and find the difference between the signals. This means that signals due to neutral particles should cancel out, and with the collector in the central position, almost no signal should be seen. If there exists an intense photon signal, it may be observed due to small misalignment of the two magnetic positions. Such a signal is observed in Fig. 8. It indicates that the photon (gamma) signal from the generator lasts at most during $25 \mathrm{~ns}$. In this experiment, the dividing wall in the chamber was $4.2 \mathrm{~mm}$ thick stainless steel, preventing most particles from penetrating the wall and reaching the collector.

In some experiments, two $\mathrm{Al}$ foils (each $20 \mu \mathrm{m}$ thick) were placed below the opening in the dividing wall in the chamber. In this way, most of the direct photon signal at low energy was eliminated. An intriguing effect of the bias voltage on the collector can then be observed. In Fig. 9, the signal in the central pin position with zero bias is shown. The time constant is $13 \mathrm{~ns}$, corresponding to charged kaons $\mathrm{K}^{ \pm}$. In Fig. 10, the only parameter that is changed from Fig. 9 is the collector bias voltage from $0 \mathrm{~V}$ to $-50 \mathrm{~V}$. This gives a longer time constant of $26 \mathrm{~ns}$, corresponding to charged pions $\pi^{ \pm}$. The difference in behavior observed is of course due to differences in the particles interacting with the collector pin. With zero bias, secondary electrons with low kinetic energy cannot leave the pin and will thus not contribute to the signal, while with negative bias, they are ejected more easily. This is directly observed in the size of the peak signal, which is almost $50 \%$ larger with negative bias. The most apparent difference in the particles reaching the pin collector concerns their kinetic energy. The main (64\%) decay of $\mathrm{K}^{ \pm}$at $12.4 \mathrm{~ns}$ forms $\mu^{ \pm}$and a $\mu$ (anti)neutrino ${ }^{37}$ with $388 \mathrm{MeV}$ excess energy in all, while the decay of $\pi^{ \pm}$at $26 \mathrm{~ns}$ forms $\mu^{ \pm}$and a $\mu$ (anti)neutrino with $33.9 \mathrm{MeV}$ excess energy, thus a much lower 


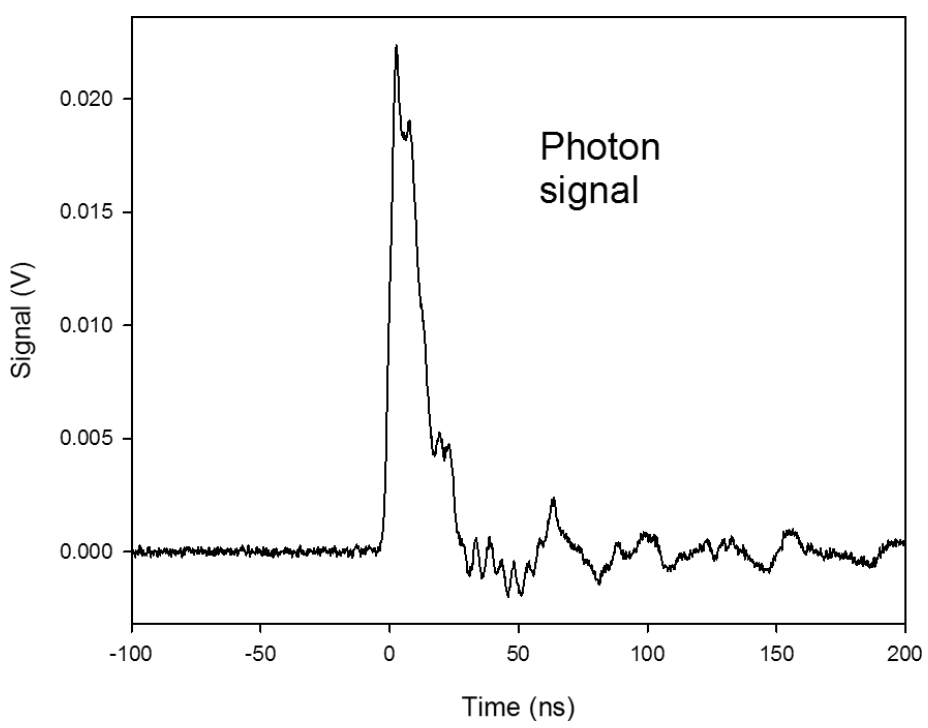

Fig. 8. Photon signal. Signal is difference normal magnet-inverted magnet, zero bias, central position no deflection.

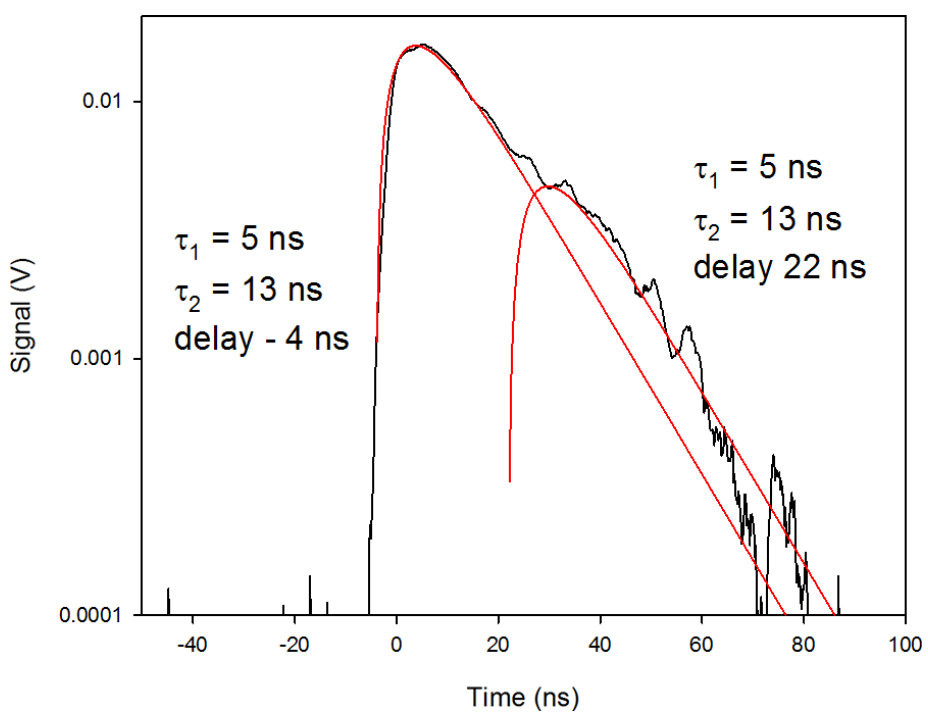

Fig. 9. Muon signal $\mu^{ \pm}$from $\mathrm{K}^{ \pm}$and delayed. Signal is difference of central no deflection-negative deflection, zero bias, no magnet, through $\mathrm{Al}$ foil.

kinetic energy. It is likely that the kinetic energy of the impinging muons on the pin collector will influence the energy of the secondary electrons formed at the pin. This means that the $\mathrm{K}^{ \pm}$decay is observed even with zero pin bias due to the high energy of the secondary electrons ejected from the pin collector, while the decay of 


\section{Holmlid}

$\pi^{ \pm}$requires an ejecting $-50 \mathrm{~V}$ bias to be seen to dominate due to the lower energy of the secondary electrons released from the pin. These effects may also have been amplified by surface processes at the pin collector due to work function changes by the applied bias. Thus, this agrees with the conclusion that the particle signal observed by the collector is due to the particles ejected by the meson decay, not due to the mesons themselves.

More complex distributions are also observed, with one interesting example in Fig. 11. No magnet was used and zero bias voltage. The first sharp peak is due to photons, similar to the peak in Fig. 8. The slower peak has a decay time-constant of $12 \mathrm{~ns}$ and a rise time $\tau_{1}$ of $10 \mathrm{~ns}$, thus it agrees with charged kaons, similar to Figs. 4 and 7. However, a broadening of the main peak is due to two similar distributions, with a delay of $10 \mathrm{~ns}$ between them. One of the directly measured signals is negative at the position of the second delayed peak, even with zero bias. This may indicate that this signal is due to particles with low energy, and that the $10 \mathrm{~ns}$ delay is due to slower transport through the apparatus. The long tail seen in the figure is close to a 52 ns decay time constant, thus a neutral kaon, but still with an initial delay indicating a slow transport.

From the figures, it is also possible to estimate the kinetic energy of the particles detected. Due to the triggering in the oscilloscope the zero time is in effect at $-3 \mathrm{~ns}$, as can be seen for example in Figs. 6 and 8. The laser pulse rise is of this size, as seen in Fig. 11. In Figs. 7 and 10, a delay of a few ns exists. In Figs. 4-6 and 9, the main signal has no delay relative to the photon signal, thus the signal is relativistic with energy of at least $100 \mathrm{MeV} \mathrm{u}^{-1}$. This energy corresponds to a velocity of $0.4 c$.

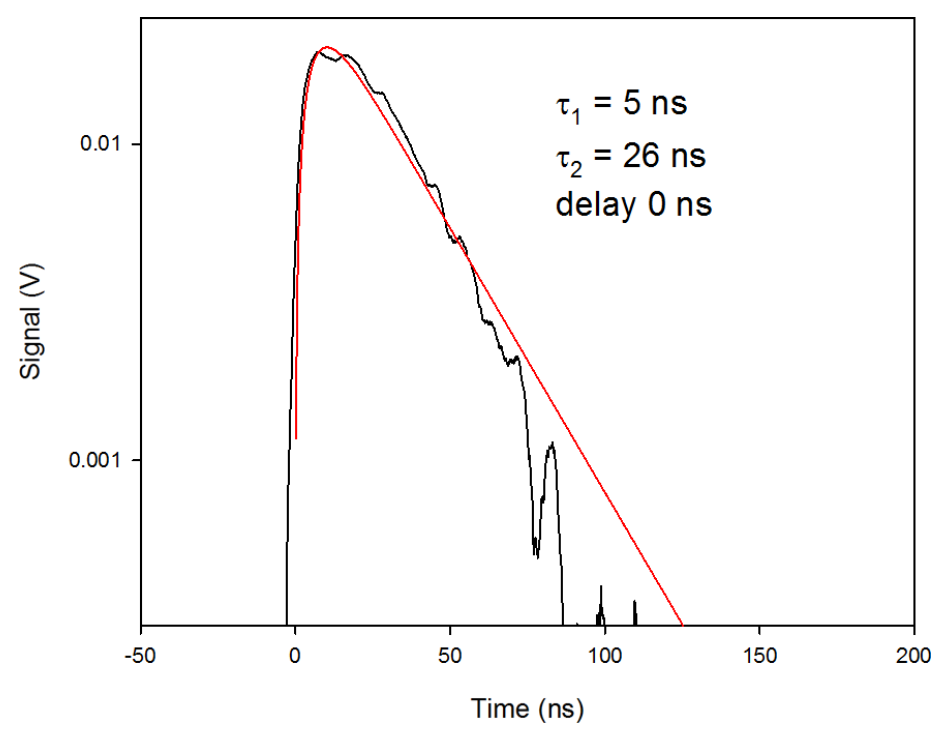

Fig. 10. Muon signal $\mu^{ \pm}$from $\pi^{ \pm}$. Signal is difference of central no deflection-negative deflection, negative bias, no magnet, through $\mathrm{Al}$ foil. 


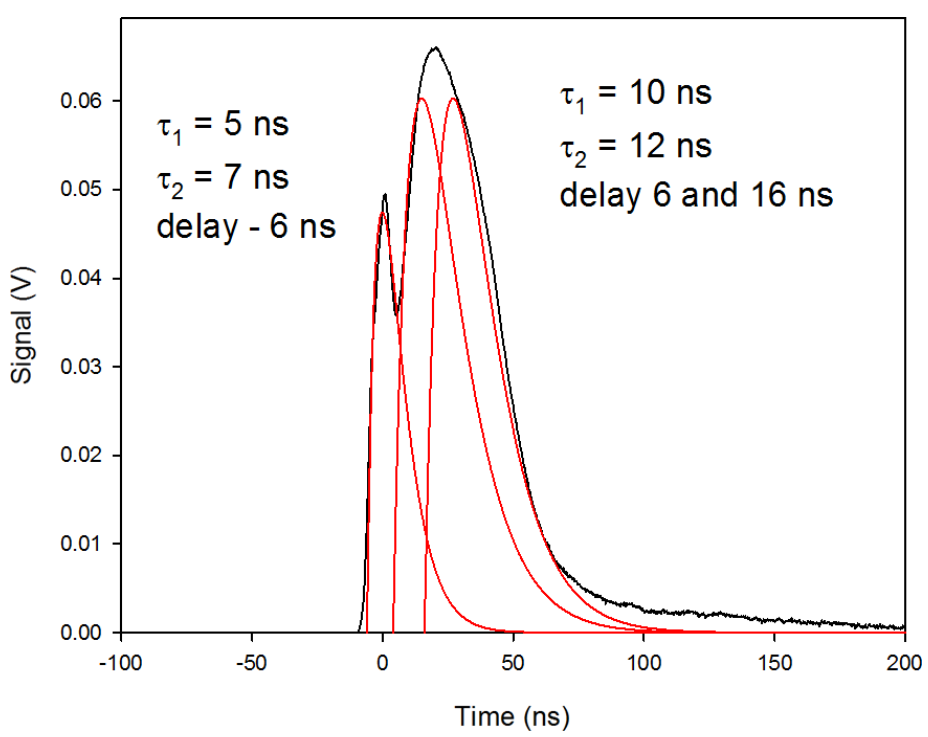

Fig. 11. Photon signal at short times and muon signal $\mu^{ \pm}$(with delays) from $\mathrm{K}^{ \pm}$. Signal is difference of negative and positive deflection, no magnet, zero bias. The deflected signal in the positive direction was partly with negative sign, giving the peak with $16 \mathrm{~ns}$ delay.

\section{Discussion}

The pulse shower of charged particles formed by the laser pulse gives a large voltage disturbance in all parts of the apparatus. In Fig. 3, the procedure used to subtract this voltage from the particle signal at the collector and to find the true particle signal to the collector is demonstrated. This disturbance is not due to the laser-pulse field or any pulsing from the laser equipment, as can be seen by increasing the gas pressure in the chamber. With a few mbar hydrogen gas, this disturbance decreases strongly. Thus, this voltage is due to the current pulse in the form of charged particles ejected from the target by the laser-pulse impact and by the countercurrent flowing in the chamber walls. Increased gas pressure stops the charged particles rapidly and the particle pulse does not give any strong current in the walls of the chamber. All the other signals shown in the figures are the signals after subtraction of the voltage disturbance measured simultaneously with another position of the collector pin.

In some of the experiments, signal delays are observed that need some discussion. The first case in Fig. 9 shows a main signal decaying with a 13 ns time constant, thus the decay of a charged kaon. The particles giving the current at the pin collector are the particles ejected from the decaying kaons, thus mainly muons. We may assume that the kaon decay means that muons with high energy are formed as in $K^{ \pm} \rightarrow \mu^{ \pm}+\nu_{\mu}$ (excess energy $388 \mathrm{MeV}$ ) with $64 \%$ probability ${ }^{37}$ and the number of kaons at the target decreases with time giving the decreasing number of muons reaching the collector. Another kaon decay channel (shown for positive kaons only) 


\section{Holmlid}

is $K^{+} \rightarrow \pi^{+}+\pi^{+}+\pi^{-}$(excess energy $75 \mathrm{MeV}$ ) with $5.6 \%$ probability. The pions formed will have relatively low kinetic energy and will decay with lifetime of $26 \mathrm{~ns}$ as $\pi^{ \pm} \rightarrow \mu^{ \pm}+\nu_{\mu}\left(\bar{\nu}_{\mu}\right)$ (excess energy $34 \mathrm{MeV}$ ). This means that the muons in this second case are not emitted directly but only after the second decay of the pions from the region in front of the target. This process will give a delayed muon signal similar to what is observed in Fig. 9. The peak intensity of this second kaon decay channel giving muons in Fig. 9 is 0.27 relative to the first channel. Since this second channel gives three pions and thus three muons per kaon, the intensity should be a factor of $3 \times 5.6 / 64=0.26$ of the first channel. This is close to the intensity observed. Another decay channel $K^{ \pm} \rightarrow \pi^{ \pm}+\pi^{0}$ with $21 \%$ probability gives $219 \mathrm{MeV}$ excess energy which will give fast pions and probably no delayed muon signal.

Another type of delay is observed in Fig. 11 as was concluded above. Transport times appear to delay both the slow tail due to neutral kaons, and also part of the main signal which is due to charged kaons. The behavior with both transport delays and decays in several steps can of course become very complex, and we just note here that such results exist without offering a complete analysis.

If the nuclear processes giving the mesons observed involves two protons at a distance of $0.6 \mathrm{pm}$ as $\mathrm{p}+\mathrm{p} \rightarrow 3 \mathrm{~K}$, the excess energy is $390 \mathrm{MeV}$. This relatively short distance exists for a time of the order of several ns, as observed experimentally. ${ }^{3}$ The two protons contain six quarks, and they are suggested to rearrange to three kaons, both charged and neutral. If the excess energy also creates a pair of muons, the remaining energy is only $180 \mathrm{MeV}$. This energy may be carried away mainly by the light muons, leaving the kaons with only low energy. The kaons may thus be almost stationary outside the target, decaying there and ejecting pions and muons with high energy. This is the situation that is observed in the experiments, with fast muons reaching the collector and showing the decay times of the kaons and pions as the decrease in time of the muon intensity reaching the collector pin.

From the analysis of the signal, it is apparent that various characteristic decay times are observed for the mesons formed at the target. Photons are also observed in the first fast peak, either with charged particles removed by the magnets as in Fig. 8 or as the first peak in an experiment with no deflecting magnetic field in Fig. 11. The signals decaying with characteristic decay times are clearly different from the photon signals.

The total energy of the muons detected at the collector is quite large. The direct energy can be found for example from Fig. 4, with a charge of $4 \times 10^{-11}$ As per laser pulse due to muons. If the muon energy is around $10 \mathrm{MeV}$, the direct energy to the collector becomes $0.4 \mathrm{~mJ}$. The total fractional area covered by the pin collector around the target is $1.4 \times 10^{-6}$. Assuming the particle intensity to be isotropic around the target, the total energy becomes $280 \mathrm{~J}$, thus a factor of 700 larger than the laser pulse energy of $<0.4 \mathrm{~J}$. The signal is probably not strictly isotropic, so the gain in energy is likely to be somewhat smaller. 


\section{Conclusions}

The results presented show that the particles moving through the magnetic field at a distance from the target are not the mesons themselves but muons formed by the decaying kaons and pions. No clear evidence is found for electrons or positrons moving from the target or close to the target, but such particles are formed closer to the collector where the particle flux is observed, probably by interaction of fast muons with the structure for example the apparatus walls. Thus, the kaons and pions formed initially have relatively low kinetic energy, while the muons normally receive an energy of at least $10 \mathrm{MeV}\left(100 \mathrm{MeV} \mathrm{u}^{-1}\right)$ in the decay.

\section{Acknowledgment}

Part of the equipment was constructed and built with support from GU Ventures $\mathrm{AB}$, The Holding Company at University of Gothenburg.

\section{References}

1. L. Holmlid, Int. J. Mass Spectrom. 351 (2013) 61.

2. S. Badiei, P. U. Andersson and L. Holmlid, Int. J. Mass Spectrom. 282 (2009) 70.

3. L. Holmlid, Int. J. Mass Spectrom. 352 (2013) 1.

4. P. U. Andersson and L. Holmlid, Phys. Lett. A 375 (2011) 1344.

5. P. U. Andersson, B. Lönn and L. Holmlid, Rev. Sci. Instrum. 82 (2011) 013503.

6. P. U. Andersson, L. Holmlid and S. R. Fuelling, J. Supercond. Nov. Mag. 25 (2012) 873.

7. L. Holmlid and S. R. Fuelling, J. Cluster Sci. 26 (2015) 1153.

8. T. Guénault, Basic Superfluids (Taylor and Francis, London, 2003).

9. S. Badiei, P. U. Andersson and L. Holmlid, Phys. Scr. 81 (2010) 045601.

10. S. Badiei, P. U. Andersson and L. Holmlid, Appl. Phys. Lett. 96 (2010) 124103.

11. L. Holmlid, Int. J. Mass Spectrom. 304 (2011) 51.

12. P. U. Andersson and L. Holmlid, Int. J. Mass Spectrom. 310 (2012) 32.

13. F. Olofson and L. Holmlid, Int. J. Mass Spectrom. 374 (2014) 33.

14. L. Holmlid, Laser Part. Beams 31 (2013) 715.

15. L. Holmlid, Int. J. Mod. Phys. E 22 (2013) 1350089.

16. L. Holmlid, Int. J. Mod. Phys. E 24 (2015) 1550026.

17. L. Holmlid, Int. J. Mod. Phys. E 24 (2015) 1550080.

18. L. W. Alvarez, H. Bradner, F. S. Crawford, Jr., J. A. Crawford, P. Falk-Vairant, M. L. Good, J. D. Gow, A. H. Rosenfeld, F. Solmitz, M. L. Stevenson, H. K. Ticho and R. D. Tripp, Phys. Rev. 105 (1957) 1127.

19. D. V. Balin, V. A. Ganzha, S. M. Kozlov, E. M. Maev, G. E. Petrov, M. A. Soroka, G. N. Schapkin, G. G. Semenchuk, V. A. Trofimov, A. A. Vasiliev, A. A. Vorobyov, N. I. Voropaev, C. Petitjean, B. Gartner, B. Lauss, J. Marton, J. Zmeskal, T. Case, K. M. Crowe, P. Kammel, F. J. Hartmann and M. P. Faifman, Phys. Part. Nuclei 42 (2011) 185.

20. L. Holmlid, J. Fusion Energy 33 (2014) 348.

21. L. Holmlid and S. Olafsson, Int. J. Hydr. Energy 40 (2015) 10559.

22. L. Holmlid, Nucl. Instrum. Methods B 296 (2013) 66.

23. P. U. Andersson and L. Holmlid, J. Fusion Energy 31 (2012) 249.

24. L. Holmlid, J. Cluster Sci. 23 (2012) 5. 
25. J. E. Hirsch, Phys. Scr. 85 (2012) 035704.

26. L. Holmlid, Chem. Phys. 237 (1998) 11.

27. L. Holmlid, Mol. Phys. 105 (2007) 933.

28. L. Holmlid, J. Mol. Struct. 885 (2008) 122.

29. A. Kamal, Particle Physics (Springer-Verlag, Berlin Heidelberg, 2014).

30. C. Nordling and J. Österman, Physics Handbook (Studentlitteratur, Lund, 1988).

31. W. E. Burcham and M. Jobes, Nuclear and Particle Physics (Pearson, Harlow, 1995).

32. G. R. Meima and P. G. Menon, Appl. Catal. A 212 (2001) 239.

33. M. Muhler, R. Schlögl and G. Ertl, J. Catal. 138 (1992) 413.

34. L. Holmlid and S. Olafsson, Int. J. Hydr. Energy 41 (2016) 1080.

35. F. Olofson and L. Holmlid, Laser Part. Beams 32 (2014) 537.

36. D. F. Measday, Phys. Rep. 354 (2001) 243.

37. Particle Data Group (C. Amsler et al.), Phys. Lett. B 667 (2008) 1. 